\title{
Complement Depletion Deteriorates Clinical Outcomes of Severe Abdominal Sepsis: A Conspirator of Infection and Coagulopathy in Crime?
}

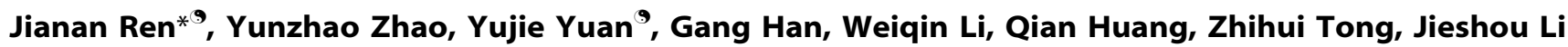 \\ Department of Surgery, Jingling Hospital, Medical School of Nanjing University, Nanjing, P. R. China
}

Abstract

Background: The complement depletion commonly occurred during sepsis, but it was often underestimated compared with severe infection or coagulation dysfunction.

Objective: This study was designed to investigate the alteration of complement system in patients with severe abdominal sepsis and evaluate the role of complement depletion in prognosis of such patients. The relationship between complement depletion and infection or coagulopathy was also explored.

Methods: Forty-five patients with severe abdominal sepsis were prospectively conducted among individuals referral to SICU. Currently recommended treatments, such as early goal-directed resuscitation, source control and antibiotics therapy, were performed. Acute physiology and chronic health evaluation II (APACHE II) and sepsis related organ failure assessment (SOFA) scores were employed to evaluate severity. Plasma levels of C3, C4, CRP, PCT, D-dimer and other parameters were detected within eight times of observation. The 28-day mortality, length of stay, and postoperative complications were compared between complement depletion and non-complement depletion groups.

Results: Within the study period, eight (17.8\%) patients died, five of them suffering from complement depletion. The overall incidence of complement depletion was $64.4 \%$. At admission, mean complement C3 and C4 levels were 0.70 and 0.13 mg/ $\mathrm{mL}$, respectively. Using ROC analysis for mortality prediction, the area under the curve of $\mathrm{C} 3$ was $0.926(95 \% \mathrm{Cl}, 0.845-0.998$, $\mathrm{P}<0.001$ ), with optimal cutpoint value of $0.578 \mathrm{mg} / \mathrm{mL}$. Complement C3 depletion was shown to be no correlation to severity scores, however, strongly correlated with elevated D-dimer, PCT concentrations and increased postoperative complications.

Conclusions: Complement C3 depletion was found to be connected to poor prognosis in severe abdominal sepsis. This depletion seems to be associated with coagulopathy and aggravated infection during sepsis, which should be paid close attention in critical care.

\section{Trial Registration: ClinicalTrials.gov NCT01568853}

Citation: Ren J, Zhao Y, Yuan Y, Han G, Li W, et al. (2012) Complement Depletion Deteriorates Clinical Outcomes of Severe Abdominal Sepsis: A Conspirator of Infection and Coagulopathy in Crime? PLoS ONE 7(10): e47095. doi:10.1371/journal.pone.0047095

Editor: Jorge I. F. Salluh, D'or Institute of Research and Education, Brazil

Received April 5, 2012; Accepted September 10, 2012; Published October 16, 2012

Copyright: ( 2012 Ren et al. This is an open-access article distributed under the terms of the Creative Commons Attribution License, which permits unrestricted use, distribution, and reproduction in any medium, provided the original author and source are credited.

Funding: This study was supported by grants from the Climbing Program in Natural Science Foundation of Jiangsu Province for Distinguished Scholars (No. BK2010017) and the High-Level Personnel Training Project, Jiangsu, China (BRA2011232). The funders had no role in study design, data collection and analysis, decision to publish, or preparation of the manuscript.

Competing Interests: The authors have declared that no competing interests exist.

*E-mail: JiananR@gmail.com

9 These authors contributed equally to this work.

\section{Introduction}

Abdominal sepsis is commonly accompanied and becomes a major problem for patients who suffered from gastrointestinal fistula [1]. Many of those patients would develop septic shock if infection from various enterogenic microorganisms was poorly uncontrolled. A typical characteristic of sepsis is the dysregulation of innate immunity, the complement system involved due to its crucial role against common pathogens [2]. In fact, the complement system does not only affect robust innate immune responses, but also plays roles in adaptive immunity regulation during the process of sepsis $[3,4]$.

There is abundant evidence for complement activation when sepsis presents. Three major pathways, including classical, lectin, and alternative, are involved to activate different biological functions, producing numerous complement anaphylatoxins (C3a, C4a, C5a, etc.) meanwhile [5]. The effects of these anaphylatoxins are still controversial, considering the protective and harmful effects in sepsis models. While there is great interest in complement by-products in human sepsis, few studies focus on the consumption of complement components and its roles in outcomes 
of sepsis. Component C3 is an indispensable community mediator for three canonical pathways of complement activation. In a way, changes of C3 levels could indirectly reflect the whole status of complement functions.

In clinical practice, most of patients with severe sepsis could develop coagulopathy very soon [6]. The activation of complement system is tightly connected with hemostasis during sepsis [7]. However, to our knowledge, few human data have investigated the detailed interconnections between complement and coagulation in severe sepsis. Furthermore, the incidence of complement depletion in severe sepsis was seldom reported, and its role in predicting mortality was also poorly understood.

Hence, the purpose of this prospective study was two-fold: first, to explore the incidence of complement depletion and its associated outcomes in a cohort of patients with severe abdominal sepsis; and second, to investigate the relationship between complement system with coagulation dysfunction and inflammation by observing the alterations of complement components and relevant parameters during the process of sepsis. All taken together serve to evaluate the application of C3 related-indexes in predicting the prognosis of such sepsis.

\section{Methods}

\section{Study Design and Ethics}

This was a prospective pilot study of a cohort of patients with severe abdominal sepsis at the Surgical Intensive Care Unit (SICU) of a tertiary level hospital in China. This facility has 37 beds, and provides critical care services for about 1500 patients each year. The period of this study was from November 2011 to March 2012.

The study was approved by the ethics committee of Jinling Hospital and registered by ClinicalTrials.gov (NCT01568853). Written informed consents were obtained from all subjects or their closest relatives. The main contents of informed consents included as follows: 1. The nature of all procedures, including the reason why the patient was suggested to join this study, when and how medicine would be prescribed and timing of sample collections; 2 . The relevant benefits, risks and uncertainties related to interventions; 3. The assessment of understanding and acceptance of interventions by the patient. The protocol for this trial is available as supporting information; see Protocol S1.

\section{Study Setting and Population}

The study cohort included patients over 18 years of age who suffered from severe abdominal sepsis. Patients would be enrolled into this study if they conformed to the following criteria: 1) confirmed diagnosis of gastrointestinal fistula through CT-scan or gastroenterography; 2) at least two of four criteria of systemic inflammatory response syndrome (SIRS) complicated with organ dysfunction, hypoperfusion (mean arterial pressure (MAP) $<60 \mathrm{mmHg}$ after $20 \mathrm{~mL} / \mathrm{kg}$ crystalloid), or hypotension (systolic blood pressure $(\mathrm{SBP})<90 \mathrm{mmHg}$ ); 3) severe intraabdominal infection, with positive culture of enterogenic microorganisms in blood or identifiable site of infection.

Detailedly, SIRS was defined by two or more of the following conditions: temperature $>38$ or $<36^{\circ} \mathrm{C}$; heart rate $>90$ beats/ min; respiratory rate $>20$ breaths $/ \mathrm{min}$ or $\mathrm{PaCO}_{2}<4.26 \mathrm{kPa}$; white blood cell count $>12,000$ or $<4,000$ cells $/ \mu \mathrm{L}$ (or $>10 \%$ immature forms). Sepsis was defined as a systemic response to infection including the criteria of SIRS plus microbiological evidence of a focal infection and/or a positive blood culture. Severe sepsis was defined as sepsis associated with organ dysfunction. Septic shock was defined by the persistent presence of sepsis-induced tissue hypoperfusion refractory to adequate fluid resuscitation. Sepsis-induced tissue hypoperfusion was characterized by $\mathrm{SBP}<90 \mathrm{mmHg}$ or $\mathrm{MAP}<70 \mathrm{mmHg}$ or a $\mathrm{SBP}$ decrease $>40 \mathrm{mmHg}$ or $<2 \mathrm{SD}$ blow normal for age in the absence of other causes of hypotension [8].

Exclusion criteria were age $<18$ or $>60$ years, pregnancy, leucopenia resulted from radiochemical therapy for malignant tumor, any primary diagnosis other than sepsis, confirmed immunodeficiency or coagulation disorders, requirement for blood transfusion (whole blood, erythrocyte, plasma and cryoprecipitate), blood purification (plasmapheresis, CRRT, GVVH, CHDF, BL, etc.), immunosuppressive agents or immediate surgery. Of note, as previously designed, patients who died within 7 days of admission were also excluded $[9,10]$.

Patients were stratified into two main groups according to the plasma levels of complement C3 after admission. Specifically, patients, who had persistent low levels of complement C3 (less than $0.75 \mathrm{mg} / \mathrm{mL}$ ) within the first three days of admission, would be categorized into complement depletion group. The rest, that included normal complement C3 or first low level but back to normal soon, would be assigned into control group. The watershed value for complement C3 was the lower limit of the reference interval in our hospital. All therapeutic interventions for sepsis were kept identical between the two groups.

\section{The Management of severe abdominal sepsis}

All enrolled patients had severe sepsis due to high volumes of fistula output or severe intra-abdominal infection from complicated appendicitis, acute pancreatitis, and other primary illnesses. The management for such populations is complicated and stageby-stage processed. In brief, the standard management of severe sepsis includes: 1. Fluid resuscitation; 2. Norepinephrine (intravenously, $10 \mu \mathrm{g} / \mathrm{min}, 2-3 \mathrm{~h}$ ) as primary vasopressor; 3. Early goaldirected therapy (MAP $>65 \mathrm{mmHg}$, and $\mathrm{SvO}_{2}>65 \%$ ); 4 . Source control; 5. Antibiotics therapy; 6. Other supporting treatments as needed. For fistula management, the four pivotal principles, outlined by Chapman and colleagues [11], consist of correction of intravascular volume deficit, drainage of abscess, control of fistula, and protection of the skin. No patient was allowed to use standardized unfractionated heparin or anticoagulant factor concentrates in the entire duration of study, without activated protein $\mathrm{G}(\mathrm{APC})$ therapy either.

In addition, nutrition therapy (parenteral/enteral nutrition), persistent surgical lavage and drainage, mechanical ventilation (ASV, GMV, IMV, etc.), somatostatin (Merck Serono, UK, 6 mg/ day) infusion and other assistant measures were selected to apply for specific patients. Short-acting insulin analogs were administrated subcutaneously to control hyperglycemia for partial cases.

\section{Complement levels and coagulation evaluation}

Samples of peripheral blood were collected into heparinized syringes (Sodium heparinate, Ratiopharm, China) at baseline, $1 \mathrm{~d}$, $2 \mathrm{~d}, 3 \mathrm{~d}, 7 \mathrm{~d}, 14 \mathrm{~d}, 21 \mathrm{~d}$, and $28 \mathrm{~d}$ of admission. Plasma was obtained from the centrifugation $\left(3000 \mathrm{~g}, 20 \mathrm{~min}, 4^{\circ} \mathrm{C}\right)$, immediately stored at $-80^{\circ} \mathrm{C}$ until tested.

The different complement components test measures were chosen according to their different baseline levels, and various sensitivities to measurement. Briefly, complement C3 was measured with the Cobas Integra platform (Roche Diagostics, Ltd., West Sussex, UK), complement C4 by nephelometry (Dade Behring, Milton Keynes, UK). Coagulation indexes, such as prothrombin time (PT), activated partial thromboplastin time (aPTT) and D-dimer, were determined at each time point of observation. Besides, other routine laboratory parameters indicat- 
ing the evolution of sepsis [white blood cells (WBC), G-reactive protein (CRP), procalcitonin (PCT), etc.] were detected meanwhile.

\section{Observing indexes and Severity Scores}

Blood routine examination, routine stool test, urine routine examination, blood biochemical analysis, blood gas analysis, drug administration (types of vasopressor, dosage, etc.) and other indexes were monitored meanwhile after admission. Those indexes were used for the simplified acute physiology score II (SAPS II), Acute Physiology and Chronic Health Evaluation II (APACHE II) score and Sequential Organ Failure Assessment (SOFA) score $[12,13]$.

\section{Outcomes and risk factors}

The primary outcome was the 28-day mortality after admission to SICU. Briefly, mortality was defined as any death occurring during the hospital stay. The predicted death rate was calculated based on the APACHE-II death equation [13]. The secondary outcomes included incidence of postoperative complications, duration of ICU stay, length of hospital stay, and hospital charges. The potential risk factors in the assessment, filtered from a univariate logistic analysis with $\mathrm{P}<0.05$ for model entry, included age, sex, body mass index $(\mathrm{BMI})$, primary disease, number of injured organs (based on International Classification of Diseases [ICD]-10 codes upon discharge), SAPS II, APACHE II, SOFA, complement C3, PCT, D-dimer and open abdomen therapy.

\section{Data Analysis and Statistics}

Data were expressed as mean \pm standard deviation if no specific statement. A Kolmogorov-Smirnov test will be used to verify the normal distribution of data. A student's $t$-test was performed for two groups' comparisons of continuous parametric variables, with the Mann-Whitney U-test for continuous variables and Fischer's exact test for categorical variables in non-parametric tests. A multivariate logistic regression using the risk factors and the outcomes (primary/secondary) was performed with stepwise method, results reported as odds ratio (OR) and 95\% confidential intervals (CIs). Cross-correlation analysis was used to detect the relationship between complement $\mathrm{C} 3$ and other parameters mentioned above, with $\mathrm{P}<0.05$ for regression significance.
Statistical analyses were performed by using SPSS Software (version 16.0; SPSS inc., Chicago, IL). Receiver operating characteristic (ROG) analysis was constructed by using STATA software (version 12.0 StataCorp., College Station, TX, USA) to evaluate the accuracy of risk prediction comparing the calculated mortality with the actual occurrence. $P$ value below 0.05 was considered statistically significant.

\section{Results}

Seventy-five patients were enrolled during the interval of this study. Generally, all enrolled patients were in accordance with community-acquired sepsis with severe illness. Of this cohort, 30 patients were excluded according to exclusion criteria, with 15 cases died within one week of admission. For those early dead, ten patients died from progressive sepsis, four from multiple organ failure, and one from uncontrolled hemorrhage. A total of 45 patients with severe sepsis were prospectively included in this study (Fig. 1). Based on baseline levels of complement C3 as mentioned above, 25 patients were assigned into complement depletion group (group 1), with the rest into control group (group 2). The general characteristics of the 45 septic patients are shown in Table 1. There was no significant difference in age, gender, BMI, severity scores, WBC, CRP and PCT levels between the two groups, but C3 and C4 baseline levels in group 1 were significantly lower than those in group 2. Besides all patients in group 1, an additional four patients had complement depletion at admission, but back to normal within three days. Hence, the incidence of complement depletion in severe sepsis was about $64.4 \%$. At the end of study, nine $(20.0 \%)$ patients from group 1 still had complement depletion.

\section{Outcomes of sepsis}

Within the period of 28-day observation, eight (17.8\%) of 45 patients died of progressive disease: five from pneumonia, two from uncontrolled intra-abdominal bleeding, and one from multiple organ failure. Except of two patients who died from pneumonia, other non-survivors suffered from severe complement C3 depletion after admission. Compared with the survivors, the dead subjects shared the similar features in respect to age, sex, and etiology, but had markedly lower baseline levels of C3 (0.36 \pm 0.18
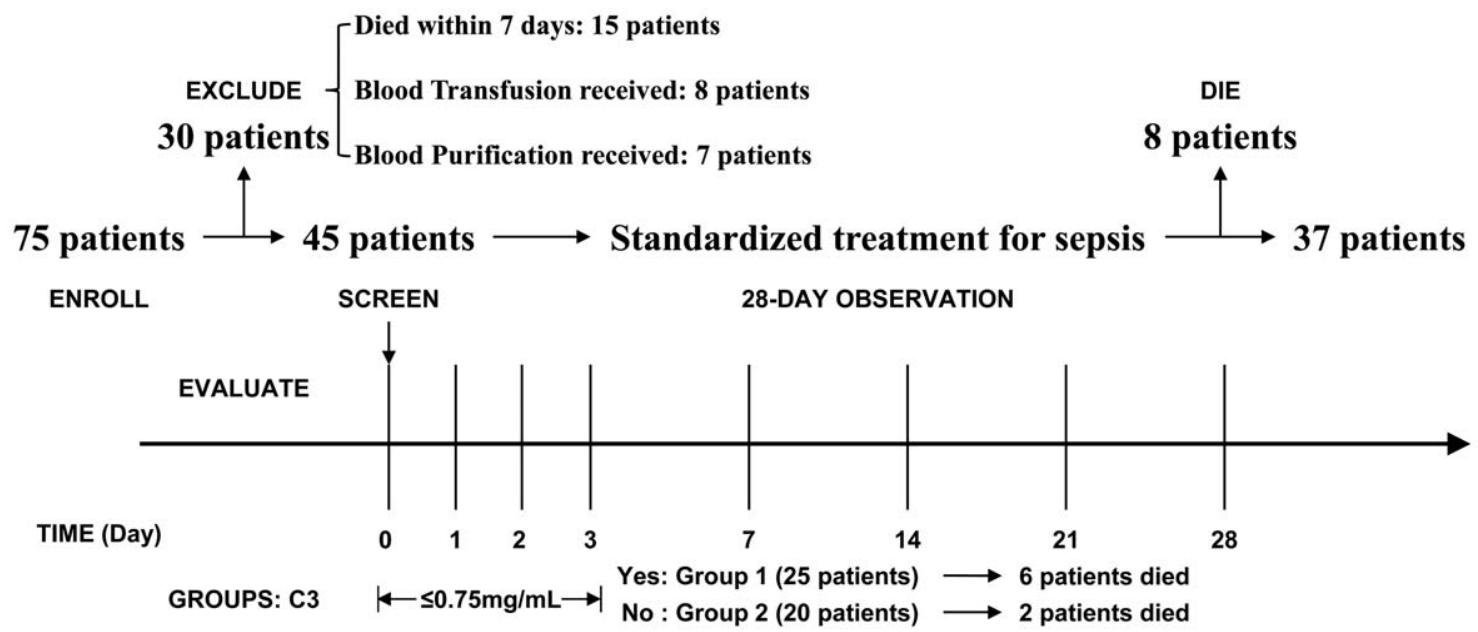

Figure 1. Enrollment and Study design. A total of eight evaluations were performed. Patients were excluded for three medical reasons, including death within seven days after admission, blood transfusion and blood purification within the period of study. All enrolled patients were followed up for 28 days, and 28-day mortality was assessed as the primary outcome.

doi:10.1371/journal.pone.0047095.g001 
Table 1. Subject's descriptive characteristics.

\begin{tabular}{|c|c|c|c|c|}
\hline Variable* & Pooled $(n=45)$ & Group $1(n=25)$ & Group $2(n=20)$ & $P^{a}$ \\
\hline Age (yr) & $43.8(13.2)$ & $44.3(11.2)$ & $43.2(10.6)$ & 0.532 \\
\hline Gender male & $82 \%$ & $80 \%$ & $85 \%$ & 0.716 \\
\hline BMI & $21.2(2.1)$ & $20.9(2.3)$ & $21.6(2.1)$ & 0.327 \\
\hline Mortality [n (\%)] & $8(18)$ & $6(24)$ & $2(10)$ & 0.269 \\
\hline \multicolumn{5}{|l|}{ Primary Disease [n] } \\
\hline SAP & 11 & 5 & 6 & 0.5 \\
\hline Selective Operation & 17 & 10 & 7 & 0.767 \\
\hline Trauma & 11 & 7 & 4 & 0.729 \\
\hline Necrotizing fasciitis & 2 & 2 & 0 & - \\
\hline Others ${ }^{b}$ & 4 & 1 & 3 & 0.309 \\
\hline APACHE II (24 h) & $17.1(1.7)$ & $17.3(1.5)$ & $16.8(1.7)$ & 0.128 \\
\hline SAPS II (24 h) & $39.7(9.1)$ & $40.2(8.9)$ & $38.2(10.3)$ & 0.088 \\
\hline SOFA (24 h) & $9.8(1.3)$ & $10.0(1.1)$ & $9.6(1.4)$ & 0.205 \\
\hline $\mathrm{C} 3(\mathrm{mg} / \mathrm{mL})$ & $0.70(0.31)$ & $0.50(0.18)$ & $0.95(0.24)$ & $<0.001$ \\
\hline $\mathrm{C} 4(\mathrm{mg} / \mathrm{mL})$ & $0.13(0.06)$ & $0.04(0.03)$ & $0.25(0.14)$ & $<0.001$ \\
\hline CRP (mg/L) & $196.1(35.7)$ & $217.3(49.6)$ & $199.5(31.3)$ & 0.074 \\
\hline PCT (ng/mL) & $11.1(2.9)$ & $11.3(2.8)$ & $10.8(3.1)$ & 0.126 \\
\hline WBC $\left(\times 10^{9} / \mathrm{L}\right)$ & $16.1(2.1)$ & $16.3(2.0)$ & $15.8(2.1)$ & 0.206 \\
\hline
\end{tabular}

*Data present as mean (SD) if no specific statement. BMI, body mass index; SAP, severe acute pancreatitis; APACHE II, acute physiology score and chronic health evaluation II; SAPS II, simplified acute physiology score II; SOFA, sequential organ failure assessment score; CRP, c-reactive protein; LOS, length of stay;

${ }^{a}$ by Mann-Whitney $U$ test or Fischer exact test;

binclude acute appendicitis, acute suppurative cholangitis, and acute gastric perforation.

doi:10.1371/journal.pone.0047095.t001

vs. $0.78 \pm 0.27, P<0.001)$, and higher APACHE II (18.1 \pm 1.1 vs. $16.2 \pm 1.7, P=0.004)$ and SOFA scores $(11.0 \pm 0.9$ vs. $9.3 \pm 1.2$, $P<0.001)$. The predicted death rate for the study was $25.0 \%$ and the observed death rate was $17.8 \%$.

Definitive operation, used to eliminate the source of infection, was performed for all patients. The mean interval from admission to a definitive operation was $16.3 \pm 6.3$ (range, 6-24) days. The difference of the interval between the two groups was significant $(21.1 \pm 10.7$ vs. $10.3 \pm 3.6$ days, $P<0.001)$.

Table 2. Outcomes after 28-day observation in two groups.
The postoperative complications occurred in nine (20\%) patients during the observation period (Table 2). The incidence of complications was increased in complement depletion group compared with control group, without significant difference $(P=0.260)$. Patients were more susceptible to pneumonia than other types of complications. Although the difference of mortality in hospital or SICU was not significant, the length of stay in both hospital and SICU was markedly extended in complement depletion patients compared with control patients (Table 2). Similarly, the mean hospital charges in complement depletion

\begin{tabular}{|c|c|c|c|c|}
\hline Variable* & Pooled $(n=45)$ & Group $1(n=25)$ & Group $2(n=20)$ & $P^{a}$ \\
\hline 28-day mortality [n (\%)] & $8(18)$ & $6(24)$ & $2(10)$ & 0.269 \\
\hline ICU mortality [n (\%)] & $7(16)$ & $5(20)$ & $2(10)$ & 0.437 \\
\hline Hospital LOS (days) & $25.3(7.1)$ & $28.2(7.2)$ & $21.3(6.3)$ & 0.008 \\
\hline ICU LOS (days) & $8.2(2.4)$ & $9.7(2.6)$ & $6.3(1.1)$ & $<0.001$ \\
\hline Open abdomen [n (\%)] & $6(13)$ & $5(20)$ & $1(5)$ & 0.205 \\
\hline Postop complications [n] & 9 & 7 & 2 & 0.260 \\
\hline Surgical site infection & 4 & 3 & 1 & 0.617 \\
\hline Pneumonia & 7 & 6 & 1 & 0.112 \\
\hline Urinary tract infection & 1 & 1 & 0 & - \\
\hline Hemorrhage & 4 & 3 & 1 & 0.617 \\
\hline Incisional hernia & 1 & 1 & 0 & - \\
\hline
\end{tabular}

*Data present as mean (SD) if no specific statement. LOS, length of stay; Postop, postoperative;

aby Mann-Whitney $U$ test or Fischer exact test.

doi:10.1371/journal.pone.0047095.t002 
Table 3. Receiver operating characteristic (ROC) curve analysis in prediction of mortality.

\begin{tabular}{llll}
\hline & & & \\
\hline Variable & AUC (CI) & Cut-off value* $^{*}$ & Sn/Sp (\%) \\
\hline APACHE II score & $0.824(0.700-0.949)$ & $>16$ & $65 / 100$ \\
SOFA score & $0.853(0.732-0.974)$ & $>10$ & $86 / 62$ \\
SAPS II score & $0.823(0.692-0.953)$ & $>41$ & $81 / 75$ \\
C3 levels $(\mathrm{mg} / \mathrm{mL})$ & $0.926(0.845-0.998)$ & $<0.578$ & $78 / 100$ \\
\hline
\end{tabular}

AUC area under curve by Hanley method to calculate standard errors, Cl 95\% confidential intervals, $\mathrm{Sn} / \mathrm{Sp}$ sensitivity/specificity, APACHE acute physiology

and chronic health evaluation, SOFA sequential organ failure assessment, SAPS II simplified acute physiology score II.

*For the severity scores, higher values are predictors of mortality; but for the C3 levels, lower values are predictors of mortality.

doi:10.1371/journal.pone.0047095.t003

group were greatly higher than those in control group $(\$ 35$, $005 \pm \$ 8,667$ vs. $\$ 25,486 \pm \$ 11,810, P=0.024)$.

\section{Relationship between complement C3 depletion and severity scores}

To investigate the role of complement depletion in prediction of mortality for such patients, ROC curve analysis with conventional APACHE II, SOFA, SAPS II scores and C3 baseline levels were constructed from the predicted and observed outcome of sepsis (Table 3). The area under the curve (AUC) of complement C3 was much larger than that through any severity scores. Besides, the optimal cutpoint of C3 for predicting 28-day mortality was $0.578 \mathrm{mg} / \mathrm{mL}$, with sensitivity $78.4 \%$ and specificity $99.8 \%$.
The baseline levels of C3 are not associated with either APACHE II or SOFA scores for such populations (Fig. 2). In addition, a similar result is obtained for complement $\mathrm{C} 4$, with $\mathrm{R}^{2}=0.155$ for APACHE II score and $\mathrm{R}^{2}=0.014$ for SOFA score. In a way, the complement depletion in early stage of sepsis may be considered as an independent risk factor in predicting clinical outcome.

The changes of complement components, inflammation and coagulation indexes

As mentioned in Methods, we divided our patients into two equal groups according to the baseline levels of C3 within the first three days of admission. The complement depletion, which included C3 and C4 depletion, was almost impossible to recover back to normal once it occurred in the early phase of sepsis (Fig. 3). There was a significant difference in overall levels of complement C3 and C4 between the two groups $(P<0.05)$. The current management strategies did little contribution to concentration improvement of complement components. Briefly, the levels of CRP and PCT were decreased in both groups, but no significant difference observed (Fig. 4A). However, it seemed that the reduction of PCT concentration in complement depletion patients was delayed as compared with control patients. As for the coagulation indexes, prothrombin time and D-dimer concentration were increased in group 1 compared to group 2, with significant difference before 7 days of admission (Fig. 4B).

\section{Linear regression and Multivariate logistic regression analysis}

Linear regression analysis by stepwise method confirmed a significant relationship between complement C3 with inflammation and coagulation (Table 4). Specifically, for every unit decrease in C3 plasma level, there is a $1.56 \mathrm{mg} / \mathrm{L}$ increase in D-dimer
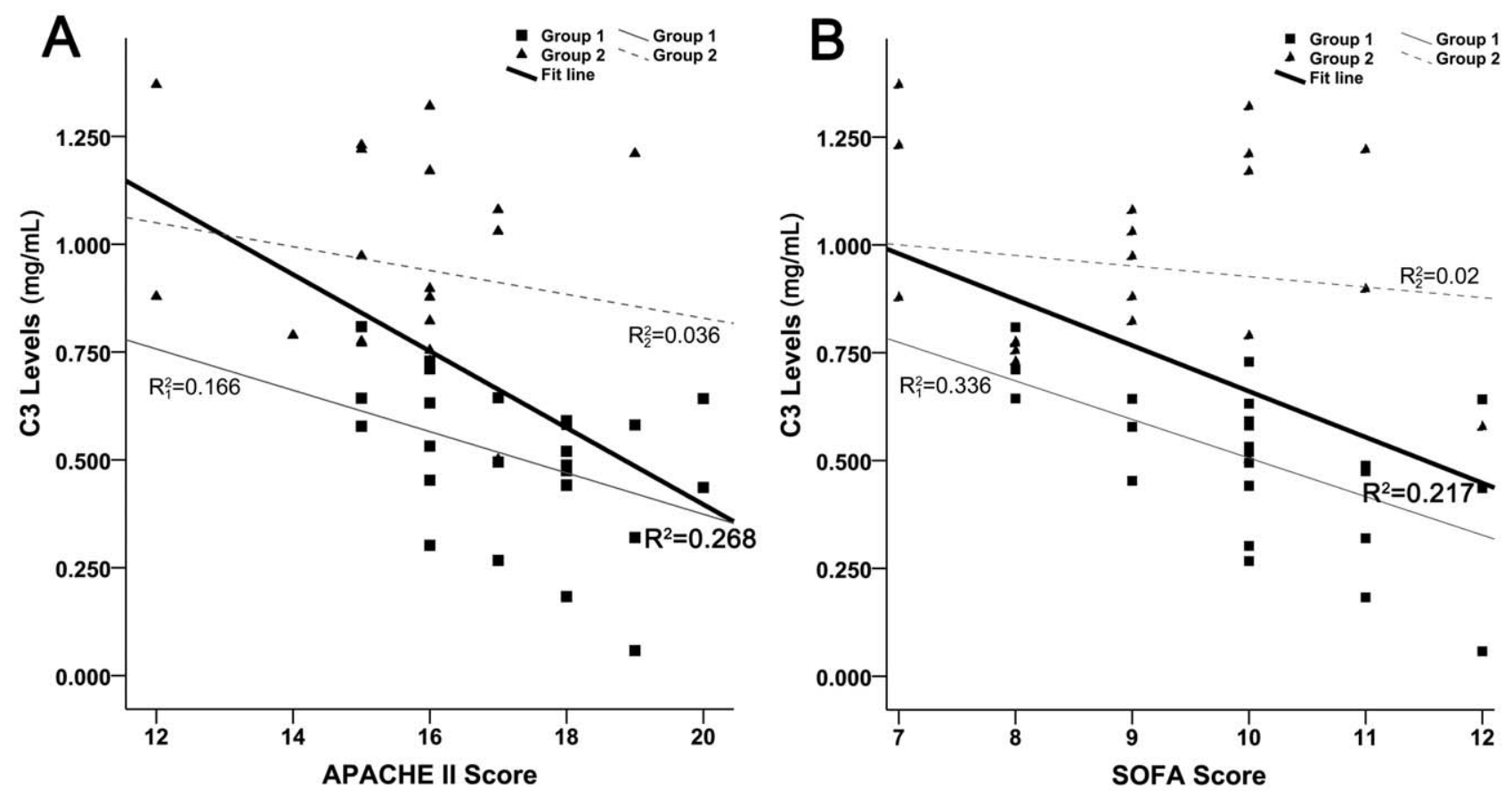

Figure 2. The correlation analysis between baseline levels of $\mathbf{C} 3$ and severity scores. (A) The relationship between $C 3$ and APACHE II score. Data in both groups were pooled for analysis $(n=45)$. The linear regression indicates that the baseline levels of C3 have little correlation with APACHE II score $\left(R^{2}=0.268\right)$. (B) The relationship between C3 and SOFA score. Data in all patients were included for analysis $(n=45)$. The regression result shows that $\mathrm{C} 3$ have no correlation with SOFA score $\left(\mathrm{R}^{2}=0.217\right)$. In addition, linear regression for each group was performed, with similar results to the pool.

doi:10.1371/journal.pone.0047095.g002 

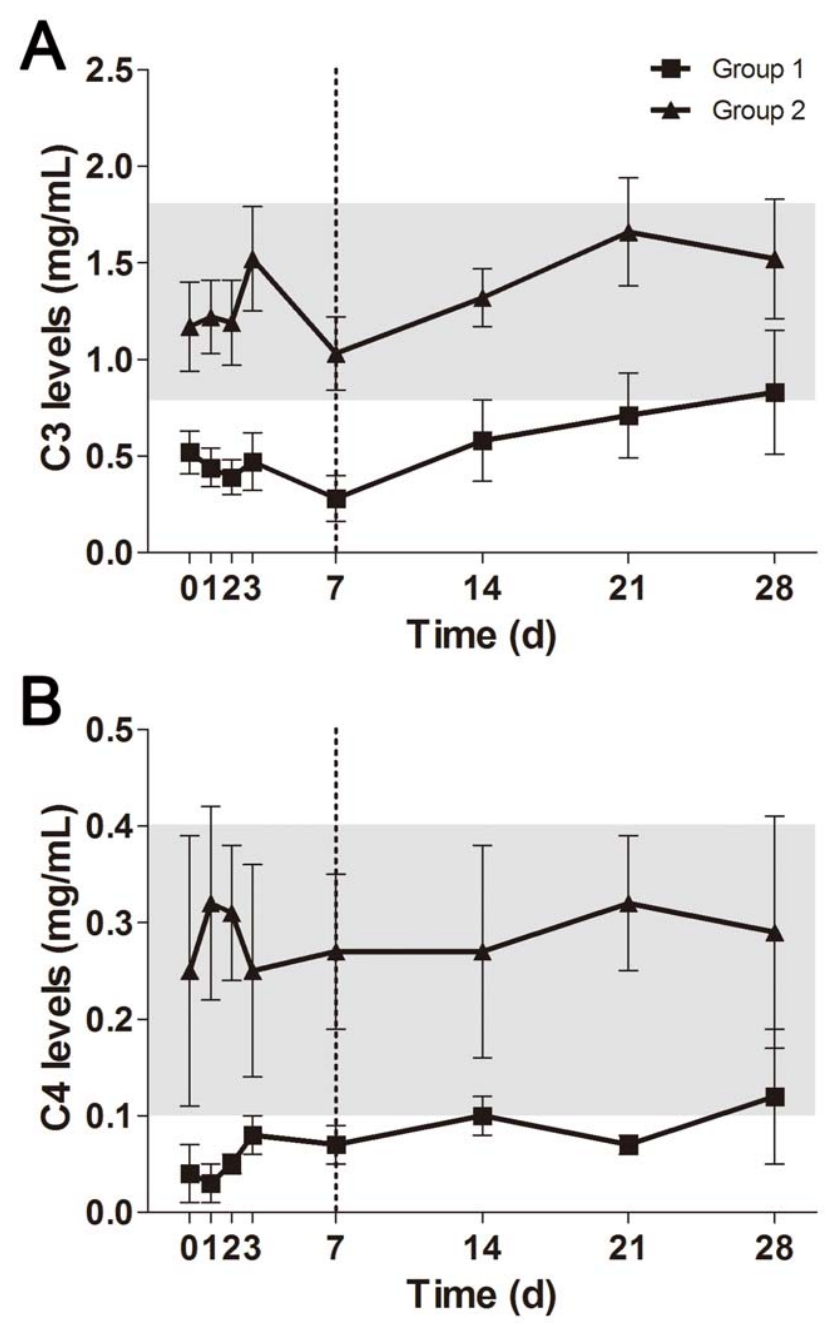

Figure 3. The observed changes of complement components in management of sepsis. These dynamic changes were directly compared between group 1 and group 2. Plasma levels of C3 (A) and C4 (B) within 28-day observation were quite different between both groups $(P<0.001)$. The gray area indicates the normal reference range. Data present as mean \pm SD.

doi:10.1371/journal.pone.0047095.g003

concentration (95\% CI, 1.13-1.98; $P<0.001)$ and a $8.5 \mathrm{ng} / \mathrm{mL}$ increase in PCT concentration (95\% CI, 6.9-10.1; $P<0.001)$, respectively. In addition, the complement depletion in early stage of sepsis was further associated with prolonged length of stay in both ICU $(P<0.0001)$ and hospital $(P<0.0001)$. Using multivariate logistic regression, we sought to identify predictors of poor outcomes of sepsis. Age, severity scores, C3 levels, and D-dimer concentration were strongly associated with the mortality. In addition, complement C3 depletion was correlated with increased odds of pneumonia and hemorrhage (Table 5). Taken together, these results indicate that the early exhaustion of complement components is strongly associated with worse outcomes in sepsis patients. Nevertheless, the slow recovery of physiologic levels of complement C3/C4 under current management strategies might explain this increased incidence of pneumonia or hemorrhage.

\section{Discussion}

In this prospective study, we stratified enrolled sepsis patients into two main groups based on baseline levels of complement C3, rather than conventional APACHE II score. The first group consisted of patients with complement C3 levels less than $0.75 \mathrm{mg} / \mathrm{mL}$, with mean levels of $0.50 \mathrm{mg} / \mathrm{mL}$ at admission to SICU. Within 28-day observation, six patients died in this group, as compared with two patients in control group. The severity scores (APACHE II, SAPS II, and SOFA) had no significant difference between the two groups. The early complement C3 depletion, as an independent risk, had better effect than classic severity scores on prediction of mortality for septic patients. Besides, the strong association between complement C3 depletion and coagulopathy was observed, with a distinct relationship to Ddimer concentration during sepsis. The exhaustion of complement components also contributed to the increased incidence of postoperative complications subsequently.

Of note, patients who underwent blood transfusion or blood purification were excluded from this study. Generally, blood transfusion, in particular fresh frozen plasma (FFP), would provide additional complement components for the recipient [14]. Although this treatment was helpful for patients with declined or depleted complement concentrations, it must conceal the real incidence of complement depletion in septic or septic shock patients [15]. Besides, there was substantial evidence that various types of blood purification could consume complement components (C3, C4, C5, etc.) during the removal of certain inflammatory cytokines [16-18].

In clinical practice, the complement depletion is often underestimated or even ignored, as compared with severe sepsis, hemorrhage, and other critical illness. In this study, the incidence of complement C3 depletion was $64.4 \%$, with $20.0 \%$ (9 patients) at the end of observation. This high incidence was simply underestimated, but related to coagulation dysfunction [19].

Coagulopathy, in particular hypocoagulability, is a common complication after severe sepsis [6]. For decades, it was generally recognized that any hypocoagulable status after sepsis was due to iatrogenic pathogenesis. In brief, hypothermia, volume dilution, and metabolic acidosis were currently accepted consensus to explain the emergence of coagulopathy. Moreover, the recent studies have indicated that the complement depletion, such as C3, $\mathrm{Clq}$ and $\mathrm{B}$ factor, is strongly associated with the incidence of coagulopathy during sepsis [20,21].

In fact, complement components contribute significantly to thrombosis by directly strengthening blood clotting properties and by augmenting the inflammatory response, which subsequently enhance coagulation reaction [22]. The most highly studied clinical condition wherein coagulation dysfunction and complement-related inflammation coexist is sepsis. As sepsis progresses, complement activation by considerable release of cytokines (TNF$\alpha$, IL-6, IL-10, etc.) produced enormous components, such as C3a, C4a, and C5a, and further consumed the pool of complement C3 and C4 [23]. As for the observed correlation between D-dimer and C3, it can be explained through the bridge of CRP. CRP directly influences several stages of sepsis via complement activation, lipid accumulation and thrombosis [24,25]. Strong correlations were confirmed in D-dimer concentrations with circulating CRP levels [26]. Besides, D-dimer alone was reported as an early prognostic marker of the evolution and complications of certain abdominal sepsis [27]. However, the concrete mechanism remains obscured, and needs some further studies in-depth to unravel their relationship.

Although APACHE II system was found to be meaningful in outcome prediction of sepsis, the diverse values of AUC found by various studies indicated it was not specific or precise enough for mortality prediction of critically ill patients [28-32]. Recent studies have demonstrated that extensive complement activation and 

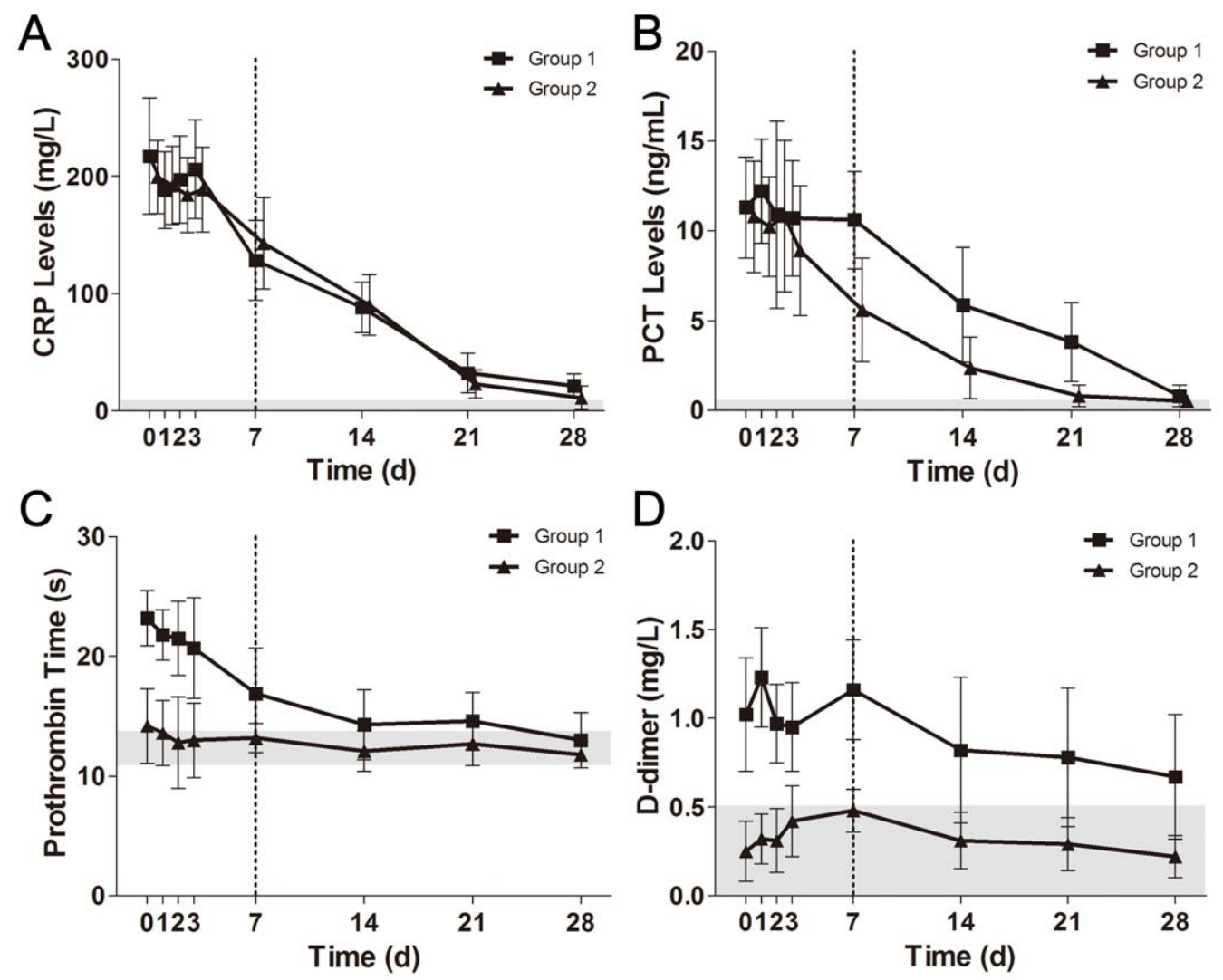

Figure 4. The observed changes of infection and coagulation indexes in management of sepsis. The values of each index were compared between group 1 and group 2. All data present as mean \pm SD. CRP (A) and PCT (B) were utilized to evaluate the inflammatory response to sepsis. PT (C) and D-dimer (D) were employed to evaluate the coagulation function during sepsis. The gray area indicates the normal reference range. doi:10.1371/journal.pone.0047095.g004

subsequent complement depletion played essential role in weakening coagulation function during sepsis [5,20]. This compromising effect would cause DIC and subsequent MODS, adversely affects the outcome of sepsis. Moreover, the exhaustion of complement C3 in late stage of sepsis was associated with significantly decreased defense to pathogenic invasion due to injured T-cell immunity [33]. Thus, complement C3 depletion could potentially reflect the severity and prognosis of septic patients. In current study, the AUC of ROG curve for mortality

Table 4. Univariate linear regression between C3 and coagulation and infection.

\begin{tabular}{llllll}
\hline Predictor & Dependent & $\mathbf{R}$ & $\mathbf{B}$ & $\mathbf{C l}$ & $P$ \\
\hline C3 & PT (s) & 0.289 & -2.153 & $-(0.128-4.179)$ & 0.038 \\
C3 & D-dimer (mg/L) & 0.722 & -1.556 & $-(1.133-1.979)$ & $<0.001$ \\
C3 & aPTT (s) & 0.319 & 3.869 & $0.601-7.137$ & 0.021 \\
C3 & Fib (mg/dL) & 0.277 & 101.6 & $1.672-201.6$ & 0.046 \\
C3 & INR (ratio) & 0.043 & -0.018 & $-0.134-0.099$ & 0.760 \\
C3 & CRP (mg/L) & 0.155 & -24.85 & $-69.86-20.17$ & 0.273 \\
C3 & PCT (ng/mL) & 0.831 & -8.543 & $-(6.918-10.16)$ & $<0.001$ \\
\hline
\end{tabular}

PT, prothrombin time; aPPT, activated partial thromboplastin time; Fib, fibrinogen; INR, international normalized ratio; CRP, c-reactive protein; PCT, procalcitonin; R, correlation coefficient; $B$, slope of the regression (also called regression coefficient). $\mathrm{Cl}$ stands for $95 \%$ confidential intervals.

doi:10.1371/journal.pone.0047095.t004 prediction was 0.926 , better than general severity scores. More importantly, our finding indicated that C3 level was not correlated with such classical severity scores, which was quite different from other prognostic biomarkers such as CRP and IL-6.

Moreover, there is also evidence indicating that the elevated PCT concentrations are associated with complement activation and depletion, in particular C3a [34]. High levels of PCT were first described in children with severe bacterial infections, and were suggested to be a specific marker for bacterial infection [35]. Importantly, the combination of increased C3a and PCT concentrations can be excellently used to discriminate patients from the sepsis to the SIRS [34].

Pulmonary neuroendocrine cells in the bronchial epithelium has been reported to be a major source of calcitonin immunoreactivity

Table 5. Complement C3 depletion and sepsis outcomes.

\begin{tabular}{llllll}
\hline Predictor & Dependent & OR & SE & CI & $P$ \\
\hline C3 & Mortality & 0.934 & 0.761 & $0.189-4.611$ & 0.034 \\
C3 & ICU mortality & 0.947 & 0.675 & $0.168-4.231$ & 0.013 \\
C3 & Pneumonia & 0.537 & 0.445 & $0.106-2.723$ & 0.006 \\
C3 & Hemorrhage & 0.255 & 0.223 & $0.046-1.414$ & 0.029 \\
\hline
\end{tabular}

Logistic regression performed by using the baseline levels of $\mathrm{C} 3$ at admission of SICU; OR, odds ratio; SE, standardized error; $\mathrm{Cl}$ stands for $95 \%$ confidential intervals.

doi:10.1371/journal.pone.0047095.t005 
$[36,37]$. In our study, the observed complement depletion may increase the risk of pulmonary infection by weakening the innate immunity. That would be reasonable to explain the destroyed structure of bronchial epithelium and its associated release of PCT into circulation. Based on our experimental results, extensive lung damages due to severe sepsis were significantly distinct, associated with the exhaustion of complement C3 [38]. However, we also found that CRP was not sensitive as PCT in accordance with complement C3 consumption, which may indicate the important role of complement in late stage of severe sepsis.

There are several limitations and questions raised by current observational study. First, the relatively small sample size would depress statistical power and bring in some uncertainty to the conclusion. The large $95 \% \mathrm{CI}$ of the ORs in Table 5 actually reflect the low number of events per covariable. Besides, another important limitation of this study is lack of generalizability for sepsis. Most of enrolled patients developed sepsis from severe intra-abdominal infection rather than other infectious sources (pulmonary infection, urinary tract infection, meningitis, etc.). Therefore, it would be insufficient to generalize the findings to sepsis at large. Finally, the complement C3a was failed to investigate since numerous studies have confirmed its extensive expression in sepsis and associated effects as a predictor for outcome of sepsis. Other complement components should be

\section{References}

1. Martinez JL, Luque-de-Leon E, Ballinas-Oseguera G, Mendez JD, JuarezOropeza MA, et al. (2012) Factors predictive of recurrence and mortality after surgical repair of enterocutaneous fistula. J Gastrointest Surg 16: 156-163; discussion 163-154.

2. Ward PA, Gao H (2009) Sepsis, complement and the dysregulated inflammatory response. J Cell Mol Med 13: 4154-4160.

3. Yalcindag A, He R, Laouini D, Alenius H, Carroll M, et al. (2006) The complement component C3 plays a critical role in both Th1 and Th2 responses to antigen. J Allergy Clin Immunol 117: 1455-1461.

4. Leendertse M, Willems RJ, Flierman R, de Vos AF, Bonten MJ, et al. (2010) The complement system facilitates clearance of Enterococcus faecium during murine peritonitis. J Infect Dis 201: 544-552.

5. Lambris JD, Hajishengallis G (2012) Innate immunity mechanisms. Adv Exp Med Biol 946: v.

6. Semeraro N, Ammollo CT, Semeraro F, Colucci M (2012) Sepsis, thrombosis and organ dysfunction. Thromb Res 129: 290-295.

7. Amara U, Flierl MA, Rittirsch D, Klos A, Chen H, et al. (2010) Molecular intercommunication between the complement and coagulation systems. J Immunol 185: 5628-5636.

8. Dellinger RP, Levy MM, Carlet JM, Bion J, Parker MM, et al. (2008) Surviving Sepsis Campaign: international guidelines for management of severe sepsis and septic shock: 2008. Crit Care Med 36: 296-327.

9. Bee TK, Croce MA, Magnotti LJ, Zarzaur BL, Maish GO 3rd, et al. (2008) Temporary abdominal closure techniques: a prospective randomized trial comparing polyglactin 910 mesh and vacuum-assisted closure. J Trauma 65: 337-342; discussion 342-334.

10. Bearfield P, Agarwal B, Ward S, Martin D, Shaw S (2010) An audit of timing of tracheostomy formation in neurosurgical patients: how long should we wait? Critical Care 14: P223.

11. Chapman R, Foran R, Dunphy JE (1964) Management of Intestinal Fistulas. Am J Surg 108: 157-164.

12. Jones AE, Trzeciak S, Kline JA (2009) The Sequential Organ Failure Assessment score for predicting outcome in patients with severe sepsis and evidence of hypoperfusion at the time of emergency department presentation. Crit Care Med 37: 1649-1654.

13. Delibegovic S, Markovic D, Hodzic S (2011) APACHE II scoring system is superior in the prediction of the outcome in critically ill patients with perforative peritonitis. Med Arh 65: 82-85.

14. Taylor R (2007) Fresh frozen plasma as a complement source. Lancet Oncol 8: $370-371$.

15. Lin RY, Astiz ME, Saxon JC, Saha DC, Rackow EC (1993) Alterations in C3, $\mathrm{C} 4$, factor $\mathrm{B}$, and related metabolites in septic shock. Clin Immunol Immunopathol 69: 136-142.

16. Peng Z, Singbartl K, Simon P, Rimmele T, Bishop J, et al. (2010) Blood purification in sepsis: a new paradigm. Contrib Nephrol 165: 322-328.

17. Iakovleva, II, Timokhov VS, Lialikova GV, Semenov VN, Moroz VV (1999) [Complement system during hemofiltration in patients with multiple organ failure]. Anesteziol Reanimatol: 23-26. tracked during sepsis to better understand the whole function of complement system. Of course, another study combining with anticoagulation therapies should be considered in future study.

In conclusion, the results of this study demonstrate that complement C3 depletion could indicate poor clinical outcomes in severe abdominal sepsis. Such depletion seems to be an independent risk factor in predicting mortality. With the small sample size, the reliable prediction is uncertain, and remains to be confirmed in further rigorous studies. Moreover, the findings suggest a correlation between C3 depletion with elevated D-dimer and PCT concentrations. The complement depletion should be paid close attention and further confirmed in critical care patients.

\section{Supporting Information}

\section{Protocol S1 Trial Protocol \\ (PDF)}

\section{Author Contributions}

Conceived and designed the experiments: JR YY. Performed the experiments: GH OH YY WL. Analyzed the data: GH YY JR YZ WL JL. Contributed reagents/materials/analysis tools: ZT GH QH YY JR. Wrote the paper: YY JR YZ. Designed the software used in analysis: YY QH ZT. Obtained permission for Clinincal trials: GH QH ZT.

18. Silvester W (1997) Mediator removal with CRRT: complement and cytokines. Am J Kidney Dis 30: S38-43.

19. Markiewski MM, Nilsson B, Ekdahl KN, Mollnes TE, Lambris JD (2007) Complement and coagulation: strangers or partners in crime? Trends Immunol 28: $184-192$.

20. Silasi-Mansat R, Zhu H, Popescu NI, Peer G, Sfyroera G, et al. (2010) Complement inhibition decreases the procoagulant response and confers organ protection in a baboon model of Escherichia coli sepsis. Blood 116: 1002-1010.

21. Ehrnthaller C, Ignatius A, Gebhard F, Huber-Lang M (2011) New insights of an old defense system: structure, function, and clinical relevance of the complement system. Mol Med 17: 317-329.

22. Oikonomopoulou K, Ricklin D, Ward PA, Lambris JD (2012) Interactions between coagulation and complement-their role in inflammation. Semin Immunopathol 34: 151-165.

23. Younger JG, Bracho DO, Chung-Esaki HM, Lee M, Rana GK, et al. (2010) Complement activation in emergency department patients with severe sepsis. Acad Emerg Med 17: 353-359.

24. Wolbink GJ, Bossink AW, Groeneveld AB, de Groot MC, Thijs LG, et al. (1998) Complement activation in patients with sepsis is in part mediated by C-reactive protein. J Infect Dis 177: 81-87.

25. Kupreishvili K, Baidoshvili A, ter Weeme M, Huybregts MA, Krijnen PA, et al. (2011) Degeneration and atherosclerosis inducing increased deposition of type IIA secretory phospholipase A2, C-reactive protein and complement in aortic valves cause neutrophilic granulocyte influx. J Heart Valve Dis 20: 29-36.

26. Yuan SM, Shi YH, Wang JJ, Lu FQ, Gao S (2011) Elevated plasma D-dimer and hypersensitive C-reactive protein levels may indicate aortic disorders. Rev Bras Cir Cardiovasc 26: 573-581.

27. Ke L, Ni HB, Tong ZH, Li WO, Li N, et al. (2012) D: -dimer as a marker of severity in patients with severe acute pancreatitis. J Hepatobiliary Pancreat Sci 19: 259-265.

28. Kulkarni SV, Naik AS, Subramanian N Jr (2007) APACHE-II scoring system in perforative peritonitis. Am J Surg 194: 549-552.

29. Zimmerman JE, Knaus WA, Judson JA, Havill JH, Trubuhovich RV, et al. (1988) Patient selection for intensive care: a comparison of New Zealand and United States hospitals. Crit Care Med 16: 318-326.

30. Sirio CA, Tajimi K, Tase C, Knaus WA, Wagner DP, et al. (1992) An initial comparison of intensive care in Japan and the United States. Crit Care Med 20: $1207-1215$.

31. Oh TE, Hutchinson R, Short S, Buckley T, Lin E, et al. (1993) Verification of the Acute Physiology and Chronic Health Evaluation scoring system in a Hong Kong intensive care unit. Crit Care Med 21: 698-705.

32. Wong DT, Crofts SL, Gomez M, McGuire GP, Byrick RJ (1995) Evaluation of predictive ability of APACHE II system and hospital outcome in Canadian intensive care unit patients. Crit Care Med 23: 1177-1183.

33. Yuan Y, Ren J, Cao S, Zhang W, Li J (2012) Exogenous C3 protein enhances the adaptive immune response to polymicrobial sepsis through down-regulation of regulatory T cells. Int Immunopharmacol 12: 271-277.

34. Selberg O, Hecker H, Martin M, Klos A, Bautsch W, et al. (2000 Discrimination of sepsis and systemic inflammatory response syndrome by 
determination of circulating plasma concentrations of procalcitonin, protein complement 3a, and interleukin-6. Crit Care Med 28: 2793-2798.

35. Assicot M, Gendrel D, Carsin H, Raymond J, Guilbaud J, et al. (1993) High serum procalcitonin concentrations in patients with sepsis and infection. Lancet 341: 515-518.

36. Yealy DM, Fine MJ (2009) Measurement of serum procalcitonin: a step closer to tailored care for respiratory infections? JAMA 302: 1115-1116.
37. Muller B, White JC, Nylen ES, Snider RH, Becker KL, et al. (2001) Ubiquitous expression of the calcitonin-i gene in multiple tissues in response to sepsis. J Clin Endocrinol Metab 86: 396-404.

38. Yuan Y, Ren J, Wu X, Cao S, Li J (2011) Exogenous C3 postpones complement exhaustion and confers organ protection in murine sepsis. J Surg Res 168: e8794. 